\title{
Trends in the Prevalence and Associated Contributing Factors of Stunting in Children Under Five Years of Age. Secondary Data Analysis of 2005, 2010 and 2014-2015 Rwanda Demographic and Health Surveys
}

\author{
Révérien Rutayisire ${ }^{1 *}$, Clémentine Kanazayire ${ }^{2}$, Germaine Tuyisenge ${ }^{3}$, Cyprien Munyanshongore ${ }^{4}$ \\ ${ }^{1}$ University of Rwanda, College of Medicine and Health Sciences, School of Health Sciences, \\ Kigali, Rwanda \\ ${ }^{2}$ University of Rwanda, College of Medicine and Health Sciences, School of Nursing and Midwifery, Kigali, Rwanda \\ ${ }^{3}$ Simon Fraser University, Department of Geography, Canada \\ ${ }^{4}$ University of Rwanda, College of Medicine and Health Sciences, School of Public Health, Kigali, Rwanda
}

*Corresponding author: Révérien Rutayisire, University of Rwanda, College of Medicine and Health Sciences, School of Health Sciences, Kigali, Rwanda. E-mail: rrutayisire@gmail.com

\begin{abstract}
Background

Stunting affects more than 161 million children under five years of age worldwide. Rwanda has a high prevalence of stunted children under five years of age ( 38\%) according to the 2014-2015 Rwanda Demographic and Health Survey.
\end{abstract}

\section{Objectives}

The aim of this study is to compare the prevalence rates of stunting in Rwanda using the Rwanda Demographic and Health Survey data of 2005, 2010 and 2014-2015.

\section{Methods}

The three Rwanda Demographic and Health Survey cross-sectional studies into consideration were conducted in 2005, 2010 and in 2014-2015. Stunting prevalence rates from those surveys were compared using Pearson's chi-squared tests and Marascuilo procedure using STATA (StataCorp. 2013. Stata Statistical Software: Release 13. College Station, TX: StataCorp LP.).

\section{Results}

The Pearson's chi-squared tests and Marascuilo procedure used in this research confirmed a significant difference between the reported three RDHS stunting prevalence rates. The trends in the stunting prevalence rates among children under five years of age showed a decrease of $13 \%$ in stunting prevalence rate, falling from $\sim 51 \%$ in 2005 to $\sim 38 \%$ in 2014-15.

\section{Conclusion}

A statistical analysis based on2005, 2010 and 2014-15 RDHS surveys datasets confirmed that there is a statistically significant reduction in stunting prevalence rates over that decade(from $\sim 51 \%$ in 2005 to $38 \%$ in 2014-2015). The main persistent associated factors with stunting were the age, sex, size at birth, residence place of the child, and the mother's educational level and household wealth index.

Keywords: Stunting; children under five years; demographic and health survey; nutrition; Rwanda 
Rwanda Journal of Medicine and Health Sciences Vol.3 No.1, March 2020 https://dx.doi.org/10.4314/rjmhs.v3i1.9

\section{INTRODUCTION}

The growth retardation known as stunting is a devastating consequence of improper nutrition in the womb, early childhood growth that may diminish a child's cognitive and physical developmental capacities[1, 2], retards linear growth[3] and leads to childhood mortality.[4, 5]Normally, stunting is measured by combining data from children's height, sex and age.[6, 7]The new World Health Organization (WHO)'s Child Growth Standards state that to be stunted, a child's height-for-age $z$-score must be less than minus two standard deviations from the median of the Child Growth Standards, among children under five years of age.[8]Stunting is not only depending on the nutrients, but it is also associated with different sociodemographic factors. Those factors are the place of residence or region,[9]wealth, mother's characteristics including age, education[10], marital status $[11,12]$ and child-related characteristics such as $\operatorname{sex}[10,13]$ age,[14] and size at birth.[14, 15]

Globally, stunting affects more than 161 million of children, and it has been reported that one-third of affected children live in Africa.[16]The Millennium Development Goals (MDs)'s reported in 2015by the United Nations[17] highlighted that stunting prevalence in children under five years of age was $24.5 \%$, and the prevalence rates of underweight and wasted children was $15 \%$ and $7.7 \%$ respectively worldwide. The prevalence of stunting of $37.8 \%$ in low and middle-income countries (LMICs) is associated with a high risk of child mortality $(\mathrm{HR}=5.48,95 \% \mathrm{CI} 4.62,6.50)$ in these countries.

In Rwanda, the prevalence of stunting shifted from $51 \%$ to $38 \%$ between 2005 and 2015 due to different efforts made by the government of Rwanda (GoR) and stakeholders.[13, 14]However, little is known about the trends and contributing factors of stunting in Rwanda. The RDHS reports delivered descriptive information regarding the proportion of the stunted population but did not show the national variability of the stunting reduction from 2005 to 2015 . The goal of this study was to describe the trends in the stunting prevalence rates in Rwanda as reported in the RDHS for 2005, 2010 and 2014-2015 surveys, and identify the persistent contributing factors.

\section{METHODS}

\section{Study design}

We used a descriptive cross-sectional design to compare stunting prevalence rates in children under five years of age from three consecutive Rwanda Demographic and Health Surveys of 2005, 2010 and 2014-15.

\section{Study area}

The study setting was Rwanda. The National Institute of Statistics of Rwanda (NISR), in collaboration with the Demographic and Health Surveys Program, collected the data for the three DHS studies. For RDHS 2005, a sample of 10,307 households was used in the survey. For RDHS 2010, 12,570 households were identified and used during the survey. In the RDHS 2014-2015, 12,717 households were selected at the time of the survey.

\section{Study population and sample size}

We used children datasets from RDHS 2005, 2010 and 2014-2015. The study population was all children under-five years of age from the selected households in the three surveys. For the 2005 RDHS, the sample size was 3,840 children, for the RDHS 2010 it was 4,133 children, for the RDHS 2014-2015 it was 3,599 children selected for completing anthropometric data.

\section{Sampling techniques}

The data used in this cross-sectional study is from the three RDHS conducted in 2005, 2010, and 2014-2015. The DHS surveys are population-based household surveys and nationally representative. The sampling frame for each DHS survey consists of households selected in a two-stage cluster sampling strategy. Selecting the primary sampling units (PSUs) was the first stage. The second stage consisted in selecting a sample of households in the enumeration areas (EA).[18-20]Households were systematically selected within clusters, and all inhabitants were enumerated. For completing a detailed questionnaire, one woman from each 
household aged 15-49 years was selected. Information related to the children within the households is contained in a data set called Children's Recode (KR). In this dataset, every child born in the five years before survey and belonging to the interviewed women had one record[21] and was eligible for height and weight measurements to determine their nutritional status.[18-20]

In each survey, RDHS collected data on every child, and, using the WHO Child Growth Standards, calculated height-for-age $z$-score (HAZ) that must be below or above the median value determined by WHO in 2006 as a growth reference population. Measurements units were centimeters, and they had to divide by a hundred in order to use meters as units of measurements. Then, stunted children were those with $z$-scores lower than -2SD.[22]

\section{Study variables}

The dependent variable for this study is stunting categorized as stunted and not stunted. This variable was computed based on the height-for-age $Z$-scores (HAZ) provided in the RDHS data sets. The explanatory variables were these: age in months, size at birth, place of residence, immunization status (vaccination) of the child; current breastfeeding status, current marital status and highest educational level of the mother, number of ANC visits during pregnancy of the mother; educational level of father or mother's partner and the wealth index of the households. All these predictor variables were categorized.

\section{Data analysis}

STATA software (StataCorp. 2013. Stata Statistical Software: Release 13. College Station, TX: StataCorpLP) was used to analyze datasets from the RDHS of 2005, 2010 and 2014-2015 surveys. To adjust for inconsistent sampling and non-response, DHS weighs its data to reestablish the sample representativeness, so that the whole sample distribution resembles to the actual population distribution of the country. When analyzing children's data, the weight variable (v005) is included in the calculations. Because there are no decimals in the DHS weight variables, we must divide by 1,000,000 before using the weight. Briefly, we created the weight variable before weighting the data.

We used the chi-square test of independence for assessing any significant relationship between stunting and predictor variables. The Marascuilo procedure was used to test the significance of the trends in stunting prevalence rates taken two-bytwo, and provided the magnitude of variation in the pairs of proportions. The null hypothesis test showed no difference between the three stunting prevalence rates. Rejecting the null hypothesis resulted in concluding that not all the proportions were equal.

The first step was to compute the differences of all pairs of proportions $\left(p_{i}-\right.$ $p_{j}$, where $i$ is not equal to $j$, and $i, j=1,2,3$ ) among all $\frac{\mathrm{k}(\mathrm{k}-1)}{2}$ pairs of proportions, $\mathrm{k}$ being the number of proportions. The absolute values of these differences are the test statistics. The second step was to choose a significance level $(\alpha=0.05)$ and compute the corresponding critical values for Marascuilo procedure[24] using the following formula:

$$
r_{i j}=\sqrt{\chi_{0.95,2}^{2}} \sqrt{\frac{p_{i}\left(1-p_{i}\right)}{n_{i}}+\frac{p_{j}\left(1-p_{j}\right)}{n_{j}}},
$$

where $\mathrm{n}_{\mathrm{i}, \mathrm{j}}$ are the sample sizes corresponding to the proportions.

The last step was to compare each of the $\frac{\mathrm{k}(\mathrm{k}-1)}{2}$ test statistics against its corresponding critical valuer $r_{i j}$. The pairs that have a test statistic that exceeds the critical value are significant at $\alpha-$ level.

The significantly associated variables with stunting were comprised in binary logistic regression to assess the persistent risk factors with stunting among the three 2005, 2010 and 2014-2015 surveys. For each of the three surveys, the backward selection method of non-significant variables was completed to get a reduced model for each RDHS. Statistically significant associated variables with stunting at a p-value less than 0.05 were kept within all adjusted models. Sampling weights were used as provided in the RDHS data sets, and were applied to all analyses. 


\section{Ethical considerations}

The permission of accessing data from the DHS program website was granted within 24 hours to access the three datasets. All surveys used by DHS program are approved by "ICF International Institutional Review Board," which guarantees that the surveys abide by the regulations of the Department of Health and Human Services of the United States human subjects' protection. DHS uses rigid measurements to keep the privacy and confidentiality of the participants and their household members. The participants were asked to accept or deny a consent form. The form informed the participants about the purpose of the survey, expected duration, procedures, potential risks and benefits along with contact information in case the participants had more questions or wanted more information. The respondents were also informed that participation were voluntary and that they could withdraw at any point. In cases where the participant is a minor, the guardian or parent must approve the consent form before the survey is completed.[25]

\section{RESULTS}

\section{Description of the study population}

Table 1 depicts the general characteristics of children under five years in 2005, 2010, 2014-15 RDHS. The same table shows that $11.57 \%$ of the 3,840 children sampled in this study were under six months in 2005, 31.06\% of them were between 6 and 23 months, and in 2014-15, most of children sampled were aged between 24 and 59 months (57.75\% of 3,599$)$. Boys sampled were $50.81 \%$ of 3,840 in $2005,50.96 \%$ of 4,133 in 2010 and $50.37 \%$ of 3,599 in 2014-15; girls sampled were $49.19 \%$ of 3,840 in $2005,49.04 \%$ of 4,133 in 2010 and $49.63 \%$ of 3,599 in 2014-15.

Among the children reported for size at birth in the three surveys, $11.54 \%$ of 4,133 were reported as very large size at birth in $2010,35.98 \%$ of 3,840 were reported as lager size than average in 2005. In $2005,46.3 \%$ of 3,840 were reported average; $13.03 \%$ of 4,133 were reported as smaller size than average at birth in $2010,2.97 \%$ of 3599 were reported as very small in 2014-15 RDHS survey. Among the three RDHS surveys, a high percentage of children were breastfed during the 2005 RDHS ( $77.37 \%$ of 3,840 sampled children) than in other RDHS surveys. High percentages were recorded for children under five who ever had a vaccination in the 2010 RDHS, with $96.79 \%$ of 4,133 sampled children. In three surveys, children sampled from rural areas were compared to their counterparts from urban areas. The frequency of less than four ANC visits was high in the 2005 RDHS (53.82\%) and that of 4 ANC visits and above was high in the 201415 RDHS (57.56\%).

In relation to marital status of the mother, the high percentages per RDHS were reported as follows: $8.82 \%$ of never-married interviewed mothers were reported in the 2014-15 RDHS; $58.72 \%$ of married women in the 2010 RDHS, $35.72 \%$ of women in cohabitation with a male reported in the 2005 RDHS; $2.44 \%$ of widowed women were reported in the 2010 RDHS , $5.04 \%$ of divorced interviewed women were reported in 2010 RDHS and $4.52 \%$ of interviewed women were reported as not living with a partner in the 2014-15 RDHS.

In terms of education, a high percentage of interviewed mothers with no education was reported in 2005 RDHS (28.34\%), 71.66\%of mothers with primary education level were reported in the 2010 RDHS, $10.54 \%$ of mothers with secondary education and $2.04 \%$ of mothers with higher education level were reported in the 2014-15 RDHS. In the 2005 RDHS, $27.19 \%$ of husbands or partners of interviewed women had primary education, whereas $71.12 \%$ had post-primary/CERAI (Centre d'Education Rurale et Artisanale Intégrée)/CERAR (Centre d'Education Rurale et Artisanale au Rwanda)/familial education level in 2014-15 RDHS; $0.96 \%$ had secondary education level in the 2005 RDHS; $3.18 \%$ had higher education in the 2014-15 RDHS. 
Rwanda Journal of Medicine and Health Sciences Vol.3 No.1, March 2020 https://dx.doi.org/10.4314/rjmhs.v3i1.9

Table 1: Characteristics of children under five years in Rwanda (2005, 2010, 2014-15 RDHS)

\begin{tabular}{|c|c|c|c|c|c|c|}
\hline \multirow{2}{*}{ Variable } & \multicolumn{2}{|c|}{ 2005(n=3840) } & \multicolumn{2}{|c|}{$2010(n=4133)$} & \multicolumn{2}{|c|}{ 2014-15(n=3599) } \\
\hline & $\mathbf{n}$ & wt. \% & $\mathbf{n}$ & wt.\% & $\mathbf{n}$ & wt. \% \\
\hline \multicolumn{7}{|l|}{ Stunting } \\
\hline Stunted & 1983 & 51.64 & 1804 & 43.65 & 1350 & 37.51 \\
\hline Not stunted & 1857 & 48.36 & 2329 & 56.35 & 2249 & 62.49 \\
\hline \multicolumn{7}{|c|}{ Child's age category in months } \\
\hline$<6$ & 446 & 11.6 & 366 & 8.8 & 353 & 9.65 \\
\hline 23-Jun & 1,205 & 31.1 & 1,214 & 29.4 & 1,171 & 32.6 \\
\hline $24-59$ & 2,189 & 57.4 & 2,553 & 61.8 & 2,091 & 57.8 \\
\hline \multicolumn{7}{|l|}{ Sex of child } \\
\hline Male & 4,379 & 50.8 & 4,586 & 51 & 3,978 & 50.4 \\
\hline Female & 4,270 & 49.2 & 4,416 & 49 & 3,878 & 49.6 \\
\hline \multicolumn{7}{|l|}{ Size of child at birth } \\
\hline Very large & 367 & 4.38 & 1,025 & 11.5 & 543 & 7.02 \\
\hline Larger than average & 3,044 & 36 & 2,986 & 33.3 & 2,511 & 32.5 \\
\hline Average & 4,112 & 46.3 & 3,547 & 39.2 & 3,497 & 44.1 \\
\hline Smaller than average & 803 & 9.55 & 1,184 & 13 & 1,026 & 12.9 \\
\hline Very small & 283 & 3.34 & 208 & 2.31 & 237 & 2.98 \\
\hline Don't know & 26 & 0.293 & 36 & 0.403 & 33 & 0.435 \\
\hline \multicolumn{7}{|l|}{ Currently breastfed } \\
\hline No & 2,029 & 22.6 & 2,575 & 28.4 & 2,376 & 30.3 \\
\hline Yes & 6,620 & 77.4 & 6,427 & 71.6 & 5,480 & 69.7 \\
\hline \multicolumn{7}{|l|}{ Ever had vaccination } \\
\hline No & 283 & 11.8 & 60 & 2.88 & 68 & 9 \\
\hline Yes & 2,033 & 86.9 & 2,000 & 96.8 & 687 & 90.6 \\
\hline \multicolumn{7}{|l|}{ Place of residence } \\
\hline Urban & 1,701 & 14.1 & 1,225 & 12 & 1,725 & 16.8 \\
\hline Rural & 6,948 & 85.9 & 7,777 & 88 & 6,131 & 83.2 \\
\hline \multicolumn{7}{|c|}{ Mother's current marital status } \\
\hline Never married & 312 & 3.36 & 536 & 5.8 & 693 & 8.66 \\
\hline
\end{tabular}


Rwanda Journal of Medicine and Health Sciences Vol.3 No.1, March 2020 https://dx.doi.org/10.4314/rjmhs.v3i1.9

$\begin{array}{lllllll}\text { Married } & 4,377 & 50.1 & 5,286 & 58.8 & 4,157 & 52.8 \\ \text { Living together } & 3,089 & 36.6 & 2,404 & 26.8 & 2,294 & 29.5 \\ \text { Widowed } & 162 & 1.81 & 220 & 2.48 & 155 & 1.96 \\ \text { Divorced } & 66 & 0.728 & 454 & 5.02 & 202 & 2.61 \\ \text { Not living together } & 643 & 7.45 & 102 & 1.11 & 355 & 4.47\end{array}$

\section{Mother's highest educational level}

$\begin{array}{lllllll}\text { No education } & 2,390 & 28.3 & 1,702 & 19.2 & 1,141 & 14.9 \\ \text { Primary } & 5,449 & 63.3 & 6,451 & 72 & 5,624 & 72.5 \\ \text { Secondary } & 762 & 7.99 & 742 & 7.85 & 891 & 10.5 \\ \text { Higher } & 48 & 0.407 & 107 & 0.93 & 200 & 2.04\end{array}$

Educational level of partner

\begin{tabular}{|c|c|c|c|c|c|c|}
\hline Primary & 2,241 & 27.2 & 1,682 & 20.1 & 1,196 & 17 \\
\hline $\begin{array}{l}\text { Post-primary/ CERAI } \\
\text { /CERAR/familial }\end{array}$ & 4,884 & 59.2 & 5,758 & 68.4 & 5,008 & 71.1 \\
\hline Secondary & 976 & 11 & 810 & 9.28 & 649 & 8.28 \\
\hline Higher & 101 & 1.02 & 156 & 1.53 & 278 & 3.18 \\
\hline Don't know & 110 & 1.41 & 57 & 0.64 & 30 & 0.46 \\
\hline \multicolumn{7}{|c|}{ Number of ANC visits during pregnancy } \\
\hline Less than 4 & 4,664 & 53.8 & 4,075 & 45.1 & 3,331 & 42.4 \\
\hline $\begin{array}{l}\text { Greater than or equal } \\
\text { to } 4\end{array}$ & 3,985 & 46.2 & 4,927 & 54.9 & 4,525 & 57.6 \\
\hline \multicolumn{7}{|l|}{ Wealth index } \\
\hline Poorest & 1,773 & 21.2 & 2,082 & 23.4 & 1,893 & 24.2 \\
\hline Poorer & 1,704 & 20.6 & 1,904 & 21.5 & 1,643 & 21.7 \\
\hline Middle & 1,676 & 20.5 & 1,767 & 19.9 & 1,479 & 19.7 \\
\hline Richer & 1,766 & 20 & 1,649 & 18.6 & 1,340 & 17.3 \\
\hline Richest & 1,730 & 17.8 & 1,600 & 16.7 & 1,501 & 17.1 \\
\hline
\end{tabular}


The household wealth index results showed a high percentage of poorest households in 2014-15 RDHS (24.19\%); the frequency of poorer households in 2014-15 RDHS was 21.7\%; for middle income households in 2005 RDHS it was $20.48 \%$;for richer households in 2005 RDHS it was $19.99 \%$ and for richest households in 2005 RDHS it was $17.77 \%$.

Table 2 shows critical values and significance of the difference in the three stunting prevalence rates reported in the 2005, 2010 and 2014-15 RDHS using the Marascuilo procedure. This procedure allows comparison of the three pairs of stunting prevalence rates taken two-by-two, and a difference between two proportions is statistically significant if its value exceeds the critical range value.[24]All the compared proportions were significantly different from each other, hence there was an evidence of a statistical significance of the difference in those reported stunting prevalence rates.

Binary logistic regression analysis was performed to find out persistent determinants of child stunting during 2005, 2010 and 2014-2015 RDHS. Table 3 shows the results from the reduced models of each survey. The adjusted model of 2005 RDHS shows that children aged between 6 and 23 months $(\mathrm{OR}=$ $3.15 ; 95 \% \mathrm{CI}=[2.39-4.20])$, and children aged between 24 and 59 months $(\mathrm{OR}=4.96 ; 95 \% \mathrm{CI}=$ [3.74- 6.58]) were 3.15 and 4.96 times more likely to be stunted than their peers of under six months respectively. The child's size at birth was found as positively associated with stunting, and those children who were born very small $(\mathrm{OR}=3.91$; 95\% $\mathrm{CI}=[2.05-7.44])$ were 3.91 times likely to be stunted than those born with very large size. Rural children $(\mathrm{OR}=1.41 ; 95 \% \mathrm{CI}=[1.12-1.77])$ were 1.41 times more likely to be stunted than children from urban areas were. Children born from the poorest families $(\mathrm{OR}=2.19 ; 95 \% \mathrm{CI}=[1.63-2.94])$ were 2.19 times more likely to be stunted than those born from the richest families.

In 2010, the reduced model shows that the category of children aged between 6 and 23 months (OR= 3.93 ; 95\% CI= [2.74-5.63]) were 3.93 times more likely to be stunted than that of under 6 months of age. The category of children between 24 and 59 months of age $(\mathrm{OR}=6.01 ; 95 \% \mathrm{CI}=[4.22-8.55])$ were 6.01 times more likely to be stunted compared to the category of children aged under 6 months. Male children $(\mathrm{OR}=1.48,95 \% \mathrm{CI}=[1.29-1.70])$ were 1.48 times more likely to be stunted than to female children. Children who were born very small $(\mathrm{OR}=2.75 ; 95 \% \mathrm{CI}=[1.61-4.73])$ were found to be 2.75 times more likely to be stunted compared to those born with very large size. Rural children (OR= $1.50,95 \% \mathrm{CI}=[1.07-2.1])$ were 1.50 times more likely to be stunted compared to urban children. Children born from mothers with no education $(\mathrm{OR}=1.85 ; 95 \% \mathrm{CI}=[1.27-2.71])$ were 1.85 times more likely to be stunted than those born from mothers with secondary educational level. Children born from the poorest families $(\mathrm{OR}=2.46$; $95 \% \mathrm{CI}=$ [1.82-3.32]) were 2.46 times more likely to be stunted than those born from the richest families.

In 2014-15, results from the reduced model show that the category of children aged between 6 and 23 months $(\mathrm{OR}=5.08 ; 95 \% \mathrm{CI}=[3.38-7.62])$, and children aged between 24 and 59 months of age $(\mathrm{OR}=6.64 ; 95 \% \mathrm{CI}=[4.39-10.05])$ were 5.08 times and 6.64 times more likely to be stunted than their peers aged under six months respectively.

Male children $(\mathrm{OR}=1.59 ; 95 \% \mathrm{CI}=(1.37-1.85])$ were 1.59 times more likely to be stunted than female children. Children born with very small size $(\mathrm{OR}=2.48 ; 95 \% \mathrm{CI}=[1.30-4.70])$ were 2.48 times more likely to be stunted than those born with very large size. Children born from mothers with no education $(\mathrm{OR}=1.72 ; 95 \% \mathrm{CI}=[1.16-2.54])$ were 1.72 times more likely to be stunted than those born from mothers with secondary educational level. Children born from poorest families $(\mathrm{OR}=2.22$; 95\% CI= [1.52-3.24]) were 2.22 times more likely to be stunted than children born from richest families. 
Rwanda Journal of Medicine and Health Sciences Vol.3 No.1, March 2020 https://dx.doi.org/10.4314/rjmhs.v3i1.9

Table 2: Critical values and significance of the difference in the three stunting prevalence rates reported in the 2005, 2010 and 2014-15 RDHS

\begin{tabular}{|l|l|c|l|l|l|}
\hline Year of Survey & $\begin{array}{l}\text { Prevalence } \\
\text { of stunting }\left(\mathbf{p}_{\mathbf{i}}\right)\end{array}$ & $\begin{array}{c}\text { Absolute Differences } \\
\left|\mathbf{p}_{\mathbf{i}}-\mathbf{p}_{\mathbf{j}}\right|\end{array}$ & Value & Critical range & Significant \\
\hline 2005 & $\mathbf{p}_{\mathbf{1}}=0.5164$ & $\left|\mathbf{p}_{\mathbf{1}}-\mathbf{p}_{\mathbf{2}}\right|$ & 0.080 & 0.054 & Yes \\
\hline 2010 & $\mathbf{p}_{\mathbf{2}}=0.4365$ & $\left|\mathbf{p}_{\mathbf{2}}-\mathbf{p}_{\mathbf{3}}\right|$ & 0.061 & 0.054 & Yes \\
\hline $2014-15$ & $\mathbf{p}_{\mathbf{3}}=0.3751$ & $\left|\mathbf{p}_{\mathbf{1}}-\mathbf{p}_{\mathbf{3}}\right|$ & 0.141 & 0.054 & Yes \\
\hline
\end{tabular}

Analysis of stunting risk factors according to 2005, 2010 and 2014-2015 RDHS

Table 3. Analysis of stunting determinants among children (0-59 months) in Rwanda (2005, 2010 and 2014-15 RDHS)

\begin{tabular}{|c|c|c|c|c|c|c|c|c|c|c|c|c|}
\hline \multicolumn{5}{|c|}{$2005(n=3840)$} & \multicolumn{4}{|c|}{$2010(n=4133)$} & \multicolumn{4}{|c|}{ 2014-15(n=3599) } \\
\hline & \multicolumn{2}{|c|}{ Full model } & \multicolumn{2}{|l|}{$\begin{array}{l}\text { Reduced } \\
\text { model }\end{array}$} & \multicolumn{2}{|c|}{ Full model } & \multicolumn{2}{|l|}{$\begin{array}{l}\text { Reduced } \\
\text { model }\end{array}$} & \multicolumn{2}{|c|}{ Full model } & \multicolumn{2}{|l|}{$\begin{array}{l}\text { Reduced } \\
\text { model }\end{array}$} \\
\hline Factors & $\begin{array}{l}\text { OR } \\
(95 \% \mathrm{CI})\end{array}$ & $\begin{array}{l}\text { p- } \\
\text { val } \\
\text { ue }\end{array}$ & $\begin{array}{l}\text { OR } \\
(95 \% \mathrm{CI})\end{array}$ & $\begin{array}{l}\text { p- } \\
\text { val } \\
\text { ue }\end{array}$ & $\begin{array}{l}\text { OR } \\
(95 \% \\
\text { CI) }\end{array}$ & $\begin{array}{l}\text { p- } \\
\text { val } \\
\text { ue }\end{array}$ & $\begin{array}{l}\text { OR } \\
(95 \% \\
\text { CI) }\end{array}$ & $\begin{array}{l}\text { p- } \\
\text { val } \\
\text { ue }\end{array}$ & $\begin{array}{l}\text { OR } \\
(95 \% \\
\text { CI) }\end{array}$ & $\begin{array}{l}\text { p- } \\
\text { val } \\
\text { ue }\end{array}$ & $\begin{array}{l}\text { OR } \\
(95 \% \\
\text { CI) }\end{array}$ & $\begin{array}{l}\text { p- } \\
\text { val } \\
\text { ue }\end{array}$ \\
\hline \multicolumn{13}{|c|}{ Child's age category in months } \\
\hline$<6$ & 1 & & 1 & & 1 & & 1 & & 1 & & 1 & \\
\hline $6-23$ & $\begin{array}{l}3.05 \\
(2.33- \\
3.99)\end{array}$ & $\begin{array}{l}< \\
0.0 \\
1\end{array}$ & $\begin{array}{l}3.15(2.3 \\
9-4.20)\end{array}$ & $\begin{array}{l}< \\
0.0 \\
1\end{array}$ & $\begin{array}{l}3.35(2.4 \\
2-4.64)\end{array}$ & $\begin{array}{l}<0 \\
01\end{array}$ & $\begin{array}{l}3.93(2.7 \\
4-5.63)\end{array}$ & $\begin{array}{l}<0 \\
01\end{array}$ & $\begin{array}{l}4.89(3.3 \\
2-7.20)\end{array}$ & $\begin{array}{l}<0 \\
01\end{array}$ & $\begin{array}{l}5.08(3.3 \\
8-7.62)\end{array}$ & $\begin{array}{l}<0 . \\
01\end{array}$ \\
\hline $24-59$ & $\begin{array}{l}4.76(3.6 \\
8-6.15)\end{array}$ & $\begin{array}{l}< \\
0.0 \\
1\end{array}$ & $\begin{array}{l}4.96(3.7 \\
4-6.58)\end{array}$ & $\begin{array}{l}< \\
0.0 \\
1\end{array}$ & $\begin{array}{l}5.14(3.7 \\
4-7.05)\end{array}$ & $\begin{array}{l}<0 \\
01\end{array}$ & $\begin{array}{l}6.01(4.2 \\
2-8.55)\end{array}$ & $\begin{array}{l}<0 \\
01\end{array}$ & $\begin{array}{l}6.48(4.3 \\
8-9.59)\end{array}$ & $\begin{array}{l}<0 \\
01\end{array}$ & $\begin{array}{l}6.64(4.3 \\
9-10.05)\end{array}$ & $\begin{array}{l}<0 . \\
01\end{array}$ \\
\hline \multicolumn{13}{|c|}{ Sex of child } \\
\hline Female & 1 & & & & 1 & & 1 & & 1 & & 1 & \\
\hline Male & $\begin{array}{l}1.1(0.96 \\
-1.27)\end{array}$ & $\begin{array}{l}0.1 \\
8\end{array}$ & & & $\begin{array}{l}1.33(1.1 \\
7-1.51)\end{array}$ & $\begin{array}{l}<0 \\
01\end{array}$ & $\begin{array}{l}1.48(1.2 \\
9-1.70)\end{array}$ & $\begin{array}{l}<0 . \\
01\end{array}$ & $\begin{array}{l}1.51(1.3 \\
1-1.73)\end{array}$ & $\begin{array}{l}<0 . \\
01\end{array}$ & $\begin{array}{l}1.59(1.3 \\
7-1.85)\end{array}$ & $\begin{array}{l}<0 . \\
01\end{array}$ \\
\hline \multicolumn{13}{|c|}{ Size of child at birth } \\
\hline $\begin{array}{l}\text { Very } \\
\text { large }\end{array}$ & 1 & $\begin{array}{l}0.0 \\
1\end{array}$ & 1 & & 1 & & 1 & & 1 & & 1 & \\
\hline $\begin{array}{l}\text { Larger } \\
\text { than } \\
\text { average }\end{array}$ & $\begin{array}{l}1.65(1.1 \\
3-2.39)\end{array}$ & $\begin{array}{l}< \\
0.0 \\
1\end{array}$ & $\begin{array}{l}1.72(1.1 \\
5-2.58)\end{array}$ & $\begin{array}{l}< \\
0.0 \\
1\end{array}$ & $\begin{array}{l}1.18(0.9 \\
4-1.47)\end{array}$ & $\begin{array}{l}1 . \\
47\end{array}$ & $\begin{array}{l}1.15(0.9 \\
0-1.47)\end{array}$ & $\begin{array}{l}0 . \\
9\end{array}$ & $\begin{array}{l}0.99(0.7 \\
2-1.37)\end{array}$ & $\begin{array}{l}0 . \\
95\end{array}$ & $\begin{array}{l}1.00(0.7 \\
1-1.40)\end{array}$ & $\begin{array}{l}0 . \\
98\end{array}$ \\
\hline
\end{tabular}


Rwanda Journal of Medicine and Health Sciences Vol.3 No.1, March 2020 https://dx.doi.org/10.4314/rjmhs.v3i1.9

\begin{tabular}{|c|c|c|c|c|c|c|c|c|c|c|c|}
\hline Average & $\begin{array}{l}1.74(1.1 \\
9-2.55)\end{array}$ & $\begin{array}{l}< \\
0.0 \\
1\end{array}$ & $\begin{array}{l}1.96(1.3 \\
0-2.96)\end{array}$ & $\begin{array}{l}< \\
0.0 \\
1\end{array}$ & $\begin{array}{l}1.22(0.9 \\
8-1.53)\end{array}$ & $\begin{array}{l}0 . \\
07\end{array}$ & $\begin{array}{l}1.35(1.0 \\
6-1.73)\end{array}$ & $\begin{array}{l}0 . \\
01\end{array}$ & $\begin{array}{l}1.2(0.87 \\
-1.63)\end{array}$ & $\begin{array}{l}0 . \\
24\end{array}$ & $\begin{array}{l}1.30(0.9 \\
4-1.80)\end{array}$ \\
\hline $\begin{array}{l}\text { Smaller } \\
\text { than } \\
\text { average }\end{array}$ & $\begin{array}{l}1.77(1.1 \\
6-2.69)\end{array}$ & $\begin{array}{l}< \\
0.0 \\
1\end{array}$ & $\begin{array}{l}2.01(1.2 \\
8-3.16)\end{array}$ & $\begin{array}{l}< \\
0.0 \\
1\end{array}$ & $\begin{array}{l}1.59(1.2 \\
3-2.05)\end{array}$ & $\begin{array}{l}<0 \\
01\end{array}$ & $\begin{array}{l}1.88(1.4 \\
2-2.48)\end{array}$ & $\begin{array}{l}<0 . \\
01\end{array}$ & $\begin{array}{l}2.06(1.4 \\
5-2.94)\end{array}$ & $\begin{array}{l}<0 \\
01\end{array}$ & $\begin{array}{l}2.24(1.5 \\
3-3.30)\end{array}$ \\
\hline $\begin{array}{l}\text { Very } \\
\text { small }\end{array}$ & $\begin{array}{l}3.70(2.0 \\
3-6.76)\end{array}$ & $\begin{array}{l}0.0 \\
5\end{array}$ & $\begin{array}{l}3.91(2.0 \\
5-7.44)\end{array}$ & $\begin{array}{l}< \\
0.0 \\
1\end{array}$ & $\begin{array}{l}2.51(1.5 \\
8-4)\end{array}$ & $\begin{array}{l}<0 \\
01\end{array}$ & $\begin{array}{l}2.75(1.6 \\
1-4.73)\end{array}$ & $\begin{array}{l}<0 . \\
01\end{array}$ & $\begin{array}{l}3.04(1.8 \\
4-5.03)\end{array}$ & $\begin{array}{l}<0 . \\
01\end{array}$ & $\begin{array}{l}2.48(1.3 \\
0-4.70)\end{array}$ \\
\hline $\begin{array}{l}\text { Don't } \\
\text { know }\end{array}$ & $\begin{array}{l}6.42(0.9 \\
9-41.49)\end{array}$ & $\begin{array}{l}0.2 \\
1\end{array}$ & $\begin{array}{l}7.87(0.9 \\
3-66.32)\end{array}$ & $\begin{array}{l}0.0 \\
6\end{array}$ & $\begin{array}{l}1.29(0.5 \\
2-3.18)\end{array}$ & $\begin{array}{l}0 . \\
58\end{array}$ & $\begin{array}{l}1.1(0.46 \\
-2.60)\end{array}$ & $\begin{array}{l}0 . \\
83\end{array}$ & 3.24 & $\begin{array}{l}0 . \\
03\end{array}$ & $\begin{array}{l}2.00(0.5 \\
2-7.69)\end{array}$ \\
\hline
\end{tabular}

\section{Currently breastfed}

$\begin{array}{lllllll}\text { No } & 1 & & 1 & & 1 & \\ & & & 0.93(0.7 & 0 . & 0.90(0.7 & 0 . \\ \text { Yes } & 0.86(0.7 & 0.1 & 9-1.1) & 41 & 7-1.06) & 21\end{array}$

\section{Ever had vaccination}

No

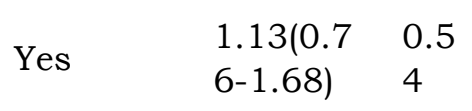

1

$1.74(0.7 \quad 0$.

$1-4.25) \quad 22$
1

$\begin{array}{ll}2.13(0.9 & 0 . \\ 0-5.01) & 08 \\ & 2\end{array}$

Place of residence

Urban

Rural

$\begin{array}{llll}1.93(1.5 & < & 1.41(1.1 & < \\ 8-2.36) & 0.0 & 2-1.77) & 0.0\end{array}$

1

1

Rural 8-2.36)

Mother's current marital status

\begin{tabular}{|c|c|c|c|c|c|}
\hline $\begin{array}{l}\text { Never } \\
\text { married }\end{array}$ & 1 & & 1 & & 1 \\
\hline Married & $\begin{array}{l}1.27(0.9 \\
-1.79)\end{array}$ & $\begin{array}{l}0.1 \\
7\end{array}$ & $\begin{array}{l}1.21(0.9 \\
0-1.62)\end{array}$ & $\begin{array}{l}0 . \\
19\end{array}$ & $\begin{array}{l}0.91(0.7 \\
1-1.16)\end{array}$ \\
\hline $\begin{array}{l}\text { Living } \\
\text { together }\end{array}$ & $\begin{array}{l}1.46(1.0 \\
1-2.09)\end{array}$ & $\begin{array}{l}0.0 \\
4\end{array}$ & $\begin{array}{l}1.3(0.96 \\
-1.76)\end{array}$ & $\begin{array}{l}0 . \\
09\end{array}$ & $\begin{array}{l}1.15(0.8 \\
8-1.50)\end{array}$ \\
\hline Widowed & $\begin{array}{l}1.74(0.9 \\
1-3.34)\end{array}$ & $\begin{array}{l}0.0 \\
9\end{array}$ & $\begin{array}{l}1.57(0.9 \\
8-2.51)\end{array}$ & $\begin{array}{l}0 . \\
06\end{array}$ & $\begin{array}{l}1.42(0.8 \\
2-2.45)\end{array}$ \\
\hline Divorced & $\begin{array}{l}2.19(0.9 \\
-5.33)\end{array}$ & $\begin{array}{l}0.0 \\
8\end{array}$ & $\begin{array}{l}1.09(0.7 \\
2-1.66)\end{array}$ & $\begin{array}{l}0 . \\
69\end{array}$ & $\begin{array}{l}1.21(0.7 \\
5-1.95)\end{array}$ \\
\hline $\begin{array}{l}\text { Not living } \\
\text { together }\end{array}$ & $\begin{array}{l}1.71(1.1 \\
3-2.58)\end{array}$ & $\begin{array}{l}0.0 \\
1\end{array}$ & $\begin{array}{l}1.37(0.4 \\
8-0.83)\end{array}$ & $\begin{array}{l}0 . \\
29\end{array}$ & $\begin{array}{l}1.54(1.0 \\
5-2.27)\end{array}$ \\
\hline
\end{tabular}


Rwanda Journal of Medicine and Health Sciences Vol.3 No.1, March 2020 https://dx.doi.org/10.4314/rjmhs.v3i1.9

\section{Mother's highest educational level}

\begin{tabular}{|c|c|c|c|c|c|c|c|c|c|c|}
\hline $\begin{array}{l}\text { Secondar } \\
\mathrm{y}\end{array}$ & 1 & & 1 & & 1 & & 1 & & & \\
\hline $\begin{array}{l}\text { No } \\
\text { educatio } \\
n\end{array}$ & $\begin{array}{l}1.87(1.3 \\
7-2.55)\end{array}$ & $\begin{array}{l}<0 \\
01\end{array}$ & $\begin{array}{l}3.18(2.3 \\
2-4.35)\end{array}$ & $\begin{array}{l}<0 . \\
01\end{array}$ & $\begin{array}{l}1.85(1.2 \\
7-2.71)\end{array}$ & $\begin{array}{l}<0 \\
01\end{array}$ & $\begin{array}{l}3.11(2.2 \\
8-4.24)\end{array}$ & $\begin{array}{l}<0 \\
01\end{array}$ & $\begin{array}{l}1.72(1.1 \\
6-2.54)\end{array}$ & $\begin{array}{l}<0 . \\
01\end{array}$ \\
\hline Primary & $\begin{array}{l}1.63(1.2 \\
1-1.18)\end{array}$ & $\begin{array}{l}< \\
0.0 \\
1\end{array}$ & $\begin{array}{l}2.28(1.7 \\
1-3.04)\end{array}$ & $\begin{array}{l}<0 . \\
01\end{array}$ & $\begin{array}{l}1.49(1.5 \\
7-2.09)\end{array}$ & $\begin{array}{l}0 . \\
02\end{array}$ & $\begin{array}{l}2.2(1.69 \\
-2.87)\end{array}$ & $\begin{array}{l}<0 . \\
01\end{array}$ & $\begin{array}{l}1.47(1.0 \\
4-2.08)\end{array}$ & $\begin{array}{l}0 . \\
02\end{array}$ \\
\hline Higher & $\begin{array}{l}0.20(0.0 \\
5-0.81)\end{array}$ & $\begin{array}{l}< \\
0.0 \\
1\end{array}$ & $\begin{array}{l}0.06(0- \\
0.46)\end{array}$ & $\begin{array}{l}<0 . \\
01\end{array}$ & $\begin{array}{l}0.07(0- \\
0.54)\end{array}$ & $\begin{array}{l}0 . \\
01\end{array}$ & $\begin{array}{l}0.19(0.0 \\
7-0.51)\end{array}$ & $\begin{array}{l}<0 \\
01\end{array}$ & $\begin{array}{l}0.38(0.1 \\
4-1.03)\end{array}$ & $\begin{array}{l}0 . \\
06\end{array}$ \\
\hline
\end{tabular}

\section{Educational level of partner}

Secondar

$\mathrm{y}$

1

101

$\begin{array}{lll} & & < \\ \text { Primary } & 1.55(1.1 & 0.0 \\ & 8-2.05) & 1\end{array}$

$2.46(1.8<0$.

2-3.30) $\quad 01$

$\begin{array}{llll}2.33(1.6 & <0 . & 1.19(0.8 & 0 . \\ 4-3.31) & 01 & 1-1.75) & 37 \\ & & & \end{array}$

Post-

primary/

CERAI

/CERAR

$1.49(1.1<$

/ familial

$1.84(1.4<0$.

$1.64(1.1<0 . \quad 1.01(0.7 \quad 0$.

1-2.39) 01

$\begin{array}{llll}9-2.26) & 01 & 0-1.44) & 97\end{array}$

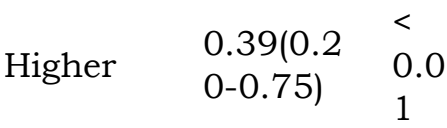

$0.76(0.2 \quad 0$.

9-2) $\quad 58$

$0.18(0.0<0 . \quad 0.37(1.1 \quad 0$.

8-0.39) $01 \quad 6-0.86) \quad 02$

Don't $\quad 1.30(0.3 \quad 0.6$

$1.36(0.6 \quad 0$.

know

$5-4.70) \quad 9$

0-3.03) $\quad 45$

$\begin{array}{llll}0.5(0.06 & 0 . & 0.40(0.0 & 0 . \\ -4.41) & 53 & 5-3.06) & 37 \\ \end{array}$

\section{Number of ANC visits during pregnancy}

Greater

than or 1

1

1

4

\begin{tabular}{|c|c|c|c|c|c|}
\hline $\begin{array}{l}\text { Less } \\
\text { than }\end{array}$ & $\begin{array}{l}1.41(1.2 \\
4-1.6)\end{array}$ & 0.0 & $\begin{array}{l}1.15(1.1 \\
9-1.30)\end{array}$ & $\begin{array}{l}0 . \\
02\end{array}$ & $\begin{array}{l}1.03(0.9 \\
0-1.19)\end{array}$ \\
\hline
\end{tabular}

Wealth index

Richest 1

1

1

1

1

1 
Rwanda Journal of Medicine and Health Sciences Vol.3 No.1, March 2020 https://dx.doi.org/10.4314/rjmhs.v3i1.9

\begin{tabular}{|c|c|c|c|c|c|c|c|c|c|c|c|c|}
\hline Poorest & $\begin{array}{l}0.53(0.4 \\
1-0.68)\end{array}$ & $\begin{array}{l}< \\
0.0 \\
1\end{array}$ & $\begin{array}{l}2.19(1.6 \\
3-2.94)\end{array}$ & $\begin{array}{l}< \\
0.0 \\
1\end{array}$ & $\begin{array}{l}3.31(2.5 \\
9-422)\end{array}$ & $\begin{array}{l}<0 . \\
01\end{array}$ & $\begin{array}{l}2.46(1.8 \\
2-3.32)\end{array}$ & $\begin{array}{l}1 . \\
82\end{array}$ & $\begin{array}{l}3.53(2.7 \\
3-4.55)\end{array}$ & $\begin{array}{l}<0 \\
01\end{array}$ & $\begin{array}{l}2.22(1.5 \\
2-3.24)\end{array}$ & $\begin{array}{l}<0 . \\
01\end{array}$ \\
\hline Poorer & $\begin{array}{l}0.53(0.4 \\
1-0.68)\end{array}$ & $\begin{array}{l}< \\
0.0 \\
1\end{array}$ & $\begin{array}{l}1.80(1.3 \\
5-2.42)\end{array}$ & $\begin{array}{l}< \\
0.0 \\
1\end{array}$ & $\begin{array}{l}3.02(2.3 \\
8-3.83)\end{array}$ & $\begin{array}{l}<0 . \\
01\end{array}$ & $\begin{array}{l}2.32(1.7 \\
4-3.10)\end{array}$ & $\begin{array}{l}<0 \\
01\end{array}$ & $\begin{array}{l}3.21(2.4 \\
7-4.19)\end{array}$ & $\begin{array}{l}<0 . \\
01\end{array}$ & $\begin{array}{l}1.87(1.2 \\
8-2.71)\end{array}$ & $\begin{array}{l}<0 . \\
01\end{array}$ \\
\hline Middle & $\begin{array}{l}0.46(0.3 \\
6-0.59)\end{array}$ & $\begin{array}{l}< \\
0.0 \\
1\end{array}$ & $\begin{array}{l}1.58(1.1 \\
7-2.13)\end{array}$ & $\begin{array}{l}< \\
0.0 \\
1\end{array}$ & $\begin{array}{l}2.38(1.8 \\
5-3.07)\end{array}$ & $\begin{array}{l}<0 . \\
01\end{array}$ & $\begin{array}{l}1.69(1.2 \\
4-2.30)\end{array}$ & $\begin{array}{l}<0 \\
01\end{array}$ & $\begin{array}{l}2.26(1.7 \\
1-2.99)\end{array}$ & $\begin{array}{l}<0 \\
01\end{array}$ & $\begin{array}{l}1.4(0.96 \\
-2.05)\end{array}$ & $\begin{array}{l}0 . \\
08\end{array}$ \\
\hline Richer & $\begin{array}{l}0.37(0.2 \\
9-0.47)\end{array}$ & $\begin{array}{l}< \\
0.0 \\
1\end{array}$ & $\begin{array}{l}1.63(1.2 \\
1-2.20)\end{array}$ & $\begin{array}{l}< \\
0.0 \\
1\end{array}$ & $\begin{array}{l}1.83(0.2 \\
8-0.41)\end{array}$ & $\begin{array}{l}<0 . \\
01\end{array}$ & $\begin{array}{l}1.37(1.0 \\
3-1.82)\end{array}$ & $\begin{array}{l}0 . \\
03\end{array}$ & $\begin{array}{l}1.6(1.21 \\
-2.1)\end{array}$ & $\begin{array}{l}<0 \\
01\end{array}$ & $\begin{array}{l}1.04(0.7 \\
2-1.51)\end{array}$ & $\begin{array}{l}0 . \\
83\end{array}$ \\
\hline
\end{tabular}




\section{DISCUSSION}

This study described the trends in the prevalence rates of stunting among Rwandan children under five years of age between 2005 and 2015 by several characteristics. The findings showed a childhood stunting reduction at the national level from $51 \%$ in 2005 to $38 \%$ in 2014-2015. This reduction of stunting prevalence rate was related to the implementation of different policies and programs developed by the Government of Rwanda.[26]

Concerning the place of residence, our findings corroborated the results from a study conducted in Mozambique and published in 2017[27],which showed that children living in urban areas had a lower risk of being stunted compared to children living in rural areas. The better complementary feeding practices, good socio-economic conditions, proper nutrition, child health improved, educated parents are mostly found in urban areas compared to the rural areas, and this could explain these findings.

Consistent with results of a study conducted in Uganda[14, 28], and other research conducted in Bangladesh[29], being born from a mother with no education was a common predictor of stunting in children under five years of age in Rwanda in 2010 and 2014-2015. The women's education contributed to teaching her accurate child feeding practices, [30] and higher education was linked with employment and higher family revenue.[11] Contrary to the African culture that gives more importance to the education of men,[31]findings from this study underscored that mother's education is as important as father's education.

Regarding the size at birth, children born with small size are at higher risk of being stunted than those born with average or larger size in the three surveys. Similar findings were reported in Bangladesh[46], in Kenya, and in Nigeria[11, 32]; poor maternal nutrition during the pregnancy could cause a small size at birth. The findings from the present study agree with other researches, such as the one conducted by Ettyang, et.al, [33] that showed that malnutrition of pregnant mothers could negatively affect child growth and development.
Results from this study showed that, in 2010 and 2014-2015, male children were more at risk of being stunted than female children of the same age under five years. Those results matched the findings in ten African countries under the Saharan desert, [34, 35] where it has been found that boys were more likely to become stunted than girls under five years of age. This can be explained by the fact that male children are more affected by adverse health outcomes than female children.[36] Consistent with findings in Nepal,[37] in comparison with children aged of less than six months, the child's age group between 6 and 59 months was associated with stunting in the final model for the three surveys.

Regarding the wealth index, the findings showed that children from poor families were significantly more likely to be stunted in 2005, 2010, and 2015 than their counterparts from rich families. Other researchers conducted in Nigeria,[38] in Bangladesh,[19]Tanzania, Kenya and Zambia,[39, 40] underscored similar findings where children from poor households were more likely to become stunted than children from rich households. This difference explains that, in comparison with poor families, rich families would have sufficient means for ensuring satisfactory nutritional consumption for children.[41]

\section{CONCLUSION}

The objective of this study was to describe the trends in the prevalence rates of under-five childhood stunting over 15 years in Rwanda and to analyze the persistent determinants of stunting. The analysis showed a statistical significant decrease of the percentage of stunted children from 2005 to 2015 in Rwanda.

The main persistent associated factors with stunting were the age, sex, size at birth, residence place of the child, educational level of mothers and household wealth index over the three 2005, 2010 and 2014-15 RDHS surveys. Combined efforts from other stakeholders in child nutrition and child health should be strengthened in order to address the high level of stunting, and factors that expose under-five children to stunting. Future studies could focus on exploring different measures that have been applied to reducing children under-five 
Rwanda Journal of Medicine and Health Sciences Vol.3 No.1, March 2020 https://dx.doi.org/10.4314/rjmhs.v3i1.9

stunting in the past decades to see the challenges to achieving higher results in this endeavor.

\section{Acknowledgements}

Financial support: The authors are thankful to the Government of Rwanda through the University of Rwanda which supported financially this study.

\section{Conflict of interest}

None.

\section{Authors' contribution}

The first author, RR, initiated the manuscript under the supervision of $\mathrm{MC}$, and $\mathrm{KC}$ and TG contributed in writing the manuscript, provided critical feedback, and helped in shaping the manuscript.

This article is published open access under the Creative Commons Attribution-

NonCommercialNoDerivatives (CC BYNC-ND4.0). People can copy and redistribute the article only for noncommercial purposes and as long as they give appropriate credit to the authors. They cannot distribute any modified material obtained by remixing, transforming or building upon this article. See https://creativecommons.org/licenses/by-ncnd/4.0/

\section{REFERENCES}

1. Dewey KG, Begum K (2011) Long-term consequences of stunting in early life. Matern Child Nutr 7:5-18

2. UNICEF (2013) Improving child nutrition: The achievable imperative for global progress. Div Commun UNICEF. doi: 978-92-8064686-3

3. Caulfield LE, Richard SA, Riverta JA, Musgrove P, Black RE (2006) Disease Control Priorities in Developing Countries. 2nd edition. Chapter 28: Stunting, Wasting and Micronutrient Deficiency Disorders. In: NCBI Bookshelf. p Chapter 28
4. Pelletier DL, Frongillo EA, Schroeder DG, Habicht JP (1995) The effects of malnutrition on child mortality in developing countries. Bull World Health Organ 73:443-8

5. De Onis M, Blössner M, Borghi E (2012) Prevalence and trends of stunting among preschool children, 1990-2020. Public Health Nutr 15:142-148

6. de Onis M (2006) WHO Child Growth Standards: Length/Height-for-Age, Weightfor-Age,Weight-for-Length, Weight-for-Height and Body Mass Index-for-Age: Methods and Development. World Heal Organ. doi: 10.4067/S0370-41062009000400012

7. Wondemeneh Tamiru M (2015) Under Nutrition and Associated Factors Among Under-Five Age Children of Kunama Ethnic Groups in Tahtay Adiyabo Woreda, Tigray Regional State, Ethiopia: Community based study. Int J Nutr Food Sci 4:277

8. Croft, Trevor N., Aileen M. J. Marshall, Courtney K. Allen et al. (2018) Guide to DHS Statistics.

9. UBOS, ICF (2012) Uganda Demograpic and Health Survey 2011. ICF Int 1-461

10. Muhoozi GKM, Atukunda P, Mwadime R, Iversen PO, Westerberg AC (2016) Nutritional and developmental status among 6- to 8month-old children in southwestern Uganda: A cross-sectional study. Food Nutr Res. doi: 10.3402/fnr.v60.30270

11. Masibo PK, Makoka D (2012) Trends and determinants of undernutrition among young Kenyan children: Kenya Demographic and Health Survey; 1993, 1998, 2003 and 20082009. Public Health Nutr 15:1715-1727

12. Habaasa G (2015) An investigation on factors associated with malnutrition among underfive children in Nakaseke and Nakasongola districts, Uganda. BMC Pediatr. doi: 10.1186/s 12887-015-0448-y

13. Willey BA, Cameron N, Norris SA, Pettifor JM, Griffiths PL (2009) Socio-economic predictors of stunting in preschool children - A population-based study from Johannesburg and Soweto. South African Med J 99:450-456 
Rwanda Journal of Medicine and Health Sciences Vol.3 No.1, March 2020 https://dx.doi.org/10.4314/rjmhs.v3i1.9

14. Kikafunda JK, Walker AF, Collett D, Tumwine JK (1998) Risk factors for early childhood malnutrition in Uganda. Pediatrics. doi: 10.1542/peds.102.4.e45

15. Fotso JC (2006) Child health inequities in developing countries: Differences across urban and rural areas. Int $\mathrm{J}$ Equity Health. doi: $10.1186 / 1475-9276-5-9$

16. United Nations Children's Fund (UNICEF), World Health Organization (WHO), The World Bank (2012) UNICEF-WHO-World Bank Joint Child Malnutrition Estimates. Nutr Rev 5:257-258

17. Senbanjo IO, Oshikoya KA, Odusanya OO, Njokanma OF (2011) Prevalence of and Risk factors for Stunting among School Children and Adole...: EBSCOhost. J Heal Popul Nutr 29:364-370

18. National Institute of Statistics of Rwanda NISR, Ministry of Health - MOH/Rwanda and II (2012) Rwanda Demographic and Health Survey 2010. Calverton, Maryland, USA

19. Institut National de la Statistique du Rwanda - INSR and ORC Macro (2006) Rwanda Demographic and Health Survey 2005.

20. INSR (2006) Rwanda 2005: Results from the Demographic and Health Survey. Stud Fam Plann 39:147-152

21. MEASURE DHS/ICF International (2019) Dataset types. https://dhsprogram.com/data/DatasetTypes.cfm.

22. Megabiaw (2013) Prevalence and Determinants of Chronic Malnutrition Among Under-5 Children in Ethiopia. Int $\mathrm{J}$ Child Heal Nutr. doi: 10.6000/19294247.2013.02.03.5

23. Wang X, Höjer B, Guo S, Luo S, Zhou W, Wang Y (2009) Stunting and "overweight" in the WHO Child Growth Standards malnutrition among children in a poor area of China. Public Health Nutr 12:1991-1998

24. Wagh ST, Razvi NA (2016) Marascuilo method of multiple comparisons (an analytical study of caesarean section delivery). Int $\mathrm{J}$ Contemp Med Res 43:2393-915
25. Karlsson E (2015) Prevalence of Stunting in Mozambique in 1997 and 2003.

26. Tom Arnold, Beckmann D (2019) Update on Scaling up Nutrition (SUN) and the "1000 Day" movements. https://www.ennonline.net/fex/41/scaling.

27. García Cruz LM, González Azpeitia G, Reyes Súarez D, Santana Rodríguez A, Loro Ferrer JF, Serra-Majem L (2017) Factors associated with stunting among children aged 0 to 59 months from the central region of Mozambique. Nutrients 9:1-16

28. Yang YY, Kaddu G, Ngendahimana D, Barkoukis H, Freedman D, Lubaale YAM, Mupere E, Bakaki PM (2018) Trends and determinants of stunting among under-5s: Evidence from the 1995, 2001, 2006 and 2011 Uganda Demographic and Health Surveys. Public Health Nutr 21:2915-2928

29. Alemu T, Umeta M (2016) Prevalence and Predictors of "Small Size" Babies in Ethiopia: In-depth Analysis of the Ethiopian Demographic and Health Survey, 2011. Ethiop J Health Sci 26:243-250

30. Nkurunziza S, Meessen B, Van J, Korachais C (2017) Determinants of stunting and severe stunting among Burundian children aged 623 months : evidence from a national crosssectional household survey , 2014. 1-14

31. Keino S, Plasqui G, Ettyang G, Van Den Borne B (2014) Determinants of stunting and overweight among young children and adolescents in sub-Saharan Africa. Food Nutr Bull 35:167-178

32. Akombi BJ, Agho KE, Hall JJ, Merom D, Astell-Burt T, Renzaho AMN (2017) Stunting and severe stunting among children under-5 years in Nigeria: A multilevel analysis. BMC Pediatr. doi: 10.1186/s12887-016-0770-z

33. Ettyang GAK, Sawe CJ (2016) Factors associated with stunting in children under age 2 in the Cambodia and Kenya 2014 Demographic and Health Surveys . DHS Work. Pap. No. 126

34. Wamani H, Åstrøm AN, Peterson S, Tumwine JK, Tylleskär T (2007) Boys are more stunted 
Rwanda Journal of Medicine and Health Sciences Vol.3 No.1, March 2020 https://dx.doi.org/10.4314/rjmhs.v3i1.9

than girls in Sub-Saharan Africa: A metaanalysis of 16 demographic and health surveys. BMC Pediatr 7:1-10

35. Wamani H, Åstrøm AN, Peterson S, Tumwine JK, Tylleskär T (2006) Predictors of poor anthropometric status among children under 2 years of age in rural Uganda. Public Health Nutr 9:320-326

36. Knaap I van der (2018) The determinants of sex differences in child stunting in Sub Saharan Africa: a multilevel logistic regression analysis. 42

37. Dorsey J, Manohar S, Neupane S, Shrestha B, Thorne-Lyman A, Webb P, Klemm RDW, West KP (2017) A multi-level risk factor assessment for stunting: Evidence from a national sample in Nepal. FASEB J. 31:
38. Black R, Alderman H, Bhutta U, et al (2013) Executive Summary of The Lancet Maternal and Child Nutrition Series. Lancet 5:1-11

39. Chirande L, Charwe D, Mbwana H, Victor R, Kimboka S, Issaka AI, Baines SK, Dibley MJ, Agho KE (2015) Determinants of stunting and severe stunting among under-fives in Tanzania: Evidence from the 2010 crosssectional household survey. BMC Pediatr. doi: 10.1186/s12887-015-0482-9

40. Hoffman D, Cacciola T, Barrios P, Simon J (2017) Temporal changes and determinants of childhood nutritional status in Kenya and Zambia. J Health Popul Nutr 36:27

41. Tiwari R, Ausman LM, Agho KE (2014) Determinants of stunting and severe stunting among under-fives: Evidence from the 2011 Nepal Demographic and Health Survey. BMC Pediatr. doi: 10.1186/1471-2431-14-239 